\title{
Analysis of the Effect of Propofol on Ovarian Function and the Level of Sex Hormone in Peripheral Blood of Mice
}

\author{
XIAOXIA QIU, XIANYUAN YI AND XIAOMIN SHI* \\ The Second People's Hospital of Gansu Province, Northwest Minda Affiliated Hospital, Lanzhou 730000, PR China
}

Qiu et al.: Analysis of the Effect of Propofol on Ovarian Function

\begin{abstract}
The mechanism of propofol action on mouse placenta time is not clear. The aim of this study was to describe the mechanism of propofol effect on ovary and uterus and to measure the level of sex hormones in peripheral blood of nonpregnant mice. On the $19^{\text {th }}$ and $20^{\text {th }} \mathrm{d}$ after luteinizing hormone peak, 14 female mice were allocated to the study group $(n=9)$ and the control group $(n=5)$, respectively and propofol and saline solution were given. On the $26^{\text {th }} \mathbf{d}$ after luteinizing hormone peak, hysterectomy was performed, to screen the concentrations of estradiol and progesterone in blood samples. Ovary, uterine horn and body were isolated for histological and morphometric diagnosis and immunohistochemical analysis of alpha estrogen and progesterone receptor expression. In the study group, progesterone decreased $(p<0.01)$ and there was no difference in the total estrogen level. There was no significant difference in histomorphology or expression of alpha estrogen and progesterone receptor in ovary. The expression of progesterone receptor was increased $(p<0.05)$ and the expression of estrogen receptor was increased $(p<0.01)(p<0.05)$. The increase of estrogen receptor may increase the sensitivity of tissue to estrogen in the blood and lead to the infiltration of monocyte in endometrium, causing obvious inflammation, hemorrhage and hyperplasia. The cornea and endometrium of female mice were significantly thicker than those of the control group $(p<0.05$ and $\mathbf{p}<0.01)$. In addition, the myometrium of the uterus was thicker than that of the angle $(p<0.001)$, while the expression of progesterone receptor in the uterus was higher $(p<0.01)$. There was no difference between cornea and body. There is no difference between propofol and other anesthetics. The types of anesthetics did not seem to affect the levels of sex hormones in the peripheral blood of mice.
\end{abstract}

Key words: Propofol, ovarian function, peripheral blood, sex hormone

Women of childbearing age and their gynaecologists are concerned about the postoperative level of female sex hormones after ovarian surgery ${ }^{[1]}$. It has been proposed that the level of super physiological or sub physiological hormones may be caused by the damage of blood supply after operation, or even by the use of specific types of anesthetics ${ }^{[2]}$. Assuming that there is no significant difference in intraoperative or intraoperative surgical skills, the question is whether specific anesthetics affect postoperative hormone levels.

General anesthesia is an essential part of surgery ${ }^{[3]}$. General anesthesia is an unrecognizable chemical drug, which can depress all excitable tissues and produce reversible unconsciousness, while the whole body has no pain $^{[4]}$. The pattern of depression is irregular and decreasing. Firstly, depression with higher cortical functions (i.e. conscious thinking, memory, motor control and sensory perception) is relative to medulla oblongata, which contains important centers of

*Address for correspondence

E-mail: 610521837@qq.com

September-October 2021 cardiovascular and respiratory system ${ }^{[5]}$. In contrast to local anesthetics, general anesthetics play a major role in the central nervous system ${ }^{[1]}$. It should also be noted that drugs may have useful anesthetic effects without good analgesic effects and vice versa. Prior to the development of effective anesthetics and painkillers, as well as blood transfusions and antibiotics, major operations were almost impossible due to severe pain, bleeding and infection. Patients are usually bound, depressed or unconscious due to hypoxia, concussion or large doses of natural central nervous system inhibitors (such as ethanol or opioids) ${ }^{[6]}$. In order to use drugs as general anesthetics, their effects must be rapid,

This is an open access article distributed under the terms of the Creative Commons Attribution-NonCommercial-ShareAlike 3.0 License, which allows others to remix, tweak, and build upon the work non-commercially, as long as the author is credited and the new creations are licensed under the identical terms

Accepted 17 October 2021

Revised 18 July 2021

Received 30 March 2020

Indian J Pharm Sci 2021;83(5):1044-1050 
effective in the process of surgery and then quickly reversible. Therefore, only those with short half-life and continuous administration are useful, but not ethanol, benzodiazepine and most barbiturates ${ }^{[7]}$.

General anesthesia is usually induced by intravenous injection of anesthetics (example (e.g.,) ultra-short acting barbiturates (e.g., thiopental sodium) and nonbarbiturates (e.g., propofol, ketamine and etomidate) and then by inhalation of gases mixed with vapor (e.g., nitrous oxide) to maintain volatile liquids (e.g., desflurane and sevoflurane) and oxygen ${ }^{[8]}$. Intravenous anesthetics are generally defined as drugs that can cause loss of consciousness in an arm brain circulation time (usually $10-20 \mu \mathrm{s}$ ) at an appropriate dose ${ }^{[4]}$. Propofol (2,6-diisopropylphenol; alkylphenol derivative) was first introduced to the market by Kay and Rolly in 1977. Because of its advantages, it is currently the most commonly used anesthetic, which largely replaces barbiturates as inducers. The incidence of side effects, i.e. nausea, vomiting and postoperative drowsiness, decreased ${ }^{[9]}$. Because its pharmacokinetics allows continuous infusion, it can also be used to maintain general anesthesia, as part of the "balanced anesthesia" program, in combination with volatile anesthetics, nitrous oxide, sedative hypnotics, neuromuscular blockers and opioids or as part of the overall intravenous anesthesia technology, usually in combination with opioids ${ }^{[10]}$. It can also be used as a hypnotic for patients undergoing mechanical ventilation and conscious sedatives, especially in routine surgery or non-invasive (e.g. radiotherapy, endoscopy and Magnetic Resonance Imaging (MRI)) surgery, because it causes less nausea and vomiting than inhaling anesthetics and it has no analgesic effect.

The empirical formula of propofol is $\mathrm{C}_{12} \mathrm{H}_{18} \mathrm{O}$ and there are two isopropyl groups on both sides of the hydroxyl group in the ortho position of the phenol ring ${ }^{[11]}$. Despite the irritating anesthesia, it can still cause amnesia and immobility ${ }^{[12]}$. Although general anesthesia is considered safe and effective, the different characteristics of anesthetics mean that the importance of the correct use of anesthetics should not be underestimated. Propofol is the most commonly used drug to induce and maintain general anesthesia. According to reports, according to the type of anesthesia, local anesthesia and general anesthesia, the results of several diseases may be different ${ }^{[13]}$.

Recently, many studies have reported that propofol concentration in ovarian follicular fluid is related to dosage and duration of anesthesia. Propofol has been reported to increase apoptosis and inhibit the invasion and migration of epithelial ovarian cancer cells. It can be assumed that propofol affects ovarian function ${ }^{[14]}$. Although propofol (2,6-diisopropylphenol) is often used as a general anesthetic for ultrasound surgery, its use in transvaginal oocyte extraction is still controversial. The dose and time-dependent damage of propofol on the fertilization ability of mouse oocytes was reported. These findings have been confirmed in two recent papers, which also show that high propofol concentration reduces the maturation rate and inhibits the division to the blastocyst stage. On the other hand, studies of In Vitro Fertilization (IVF) in humans or animals using propofol did not find harmful effects or negative results. Propofol may even be beneficial to the cleavage and fertilization rate of oocytes ${ }^{[15]}$. In a retrospective study, it was reported that there was no difference in the implantation rate or pregnancy rate when propofol was used compared with other anesthesia techniques for oocyte retrieval ${ }^{[3]}$. Some scholars have confirmed that there is no difference in pregnancy rate after propofol or after ovarian metastasis of thioglutaraldehyde via gamete. However, it has been reported that laparoscopic anterior nuclear staging after propofol, nitrous oxide anesthesia is associated with lower clinical and ongoing pregnancy rates than isoflurane, nitrous oxide anesthesia. This study evaluated the concentration of propofol in follicular fluid during general anesthesia ${ }^{[4]}$. Coetsier et al. have demonstrated that the accumulation of propofol in follicular fluid is time-dependent. Their conclusions were based on pharmacokinetic characteristics using venous blood samples.

However, it has been shown in animal studies that arterial and venous blood sampling may lead to significant differences in drug concentrations and that arterial sampling may more accurately assess distribution, elimination and pharmacodynamic effects ${ }^{[10]}$. Since the accumulation of propofol in follicular fluid may have an impact on IVF and early embryo development, which may have important clinical and ethical implications, it is considered interesting to correlate the propofol concentration in follicular fluid with arterial blood samples, as this information may further limit the amount of propofol eventually used ${ }^{[16]}$.

However, little is known about the effect of propofol on ovarian function and whether the effect of propofol in the ovary is different from that of other non-propofol anesthetics. Our aim was to investigate differences in sex hormone levels related to ovarian function based on 
the use of propofol during anesthesia. In this study, we compared postoperative Follicle Stimulating Hormone (FSH) and Estradiol (E2) levels between the control group and the group requiring anesthesia for surgery. We performed a subgroup analysis of two groups treated with propofol or non-propofol anesthetics. There were no differences between the two subgroups in baseline physical characteristics or variables related to surgery.

\section{MATERIALS AND METHODS}

\section{Animals:}

On the basis of routine clinical examination, 14 healthy female mice weighing 300-400 g from June to August were studied. According to the history of each mouse, the interval of interest in the previous cycle was normal. During the study, the animals remained in the small animal hospital of the Veterinary Medical College and were fed a diet at will. Since the first appearance of vulvar serous secretions indicates estrus, vaginal smears were collected from each mouse daily and examined rapidly with Hematoxylin-Eosin staining (H\&E) until the $1^{\text {st }} \mathrm{d}$ of cell division.

\section{Treatment regimen:}

The mice were randomly assigned to either the control group $(n=5)$ or the study group $(n=9)$. On the $19^{\text {th }}$ and $20^{\text {th }} \mathrm{d}$ after the peak of Luteinizing Hormone ( $\mathrm{LH}$ ), female mice in the control group were subcutaneously injected with saline solution $(0.3 \mathrm{ml} / \mathrm{kg}$ body weight (bw)), while female mice in the study group were subcutaneously injected with propofol (10 mg/kg bw).

\section{Blood samples:}

Blood samples $(2 \mathrm{ml})$ were collected daily from the cephalic vein into the trunk tube from d 5-7 after the onset of external symptoms of fever to detect peak LH (d 0) and then for 26 consecutive days. The LH peak (d 0) was determined based on certain Progesterone (P4) level (6.4-9.5 nmol/1) and the characteristic mesenteric cells (foams, parabasal and small intermediates) that appeared after $8 \mathrm{~d}$. Blood samples were obtained before saline or aglepristone (RU534) treatment. The blood samples were then centrifuged ( $3000 \mathrm{~g}$ for $15 \mathrm{~min}$ ) and the serum was stored at $-20^{\circ}$ until $\mathrm{P} 4$ and total estrogen (E) were determined.

\section{Hormone determination:}

To quantify $\mathrm{P} 4$ and $\mathrm{E}$, commercial immunoenzymatic assays were used. After extraction with ethyl acetate, the concentration of $\mathrm{E}$ was measured. Fluorescence was measured with a Pointe 2000 instrument. Each analysis in each series was repeated twice. The extraction efficiency fluctuated between $92 \%$ and $99 \%$. The determination sensitivity and intra series error of P4 were $0.05 \mathrm{ng} / \mathrm{ml}(0.8 \mathrm{nmol} / \mathrm{l})$ and $8.0 \%$, respectively and $\mathrm{E} 10 \mathrm{pg} / \mathrm{ml} \mathrm{(37} \mathrm{pmol} / \mathrm{l})$ and $9.6 \%$.

\section{Organizational collection:}

On the $26^{\text {th }} \mathrm{d}$ after the estimated LH peak, female mice in the control and study groups underwent selective ovarian hysterectomy under general anesthesia induced by propofol (4 mg/kg bw) and maintained isoflurane. After removal, the genital tract was thoroughly rinsed with saline. The ovaries and part of the wall of the corners of the uterus and uterus were excised and immediately placed in $10 \%$ neutral formalin buffer.

\section{Immunohistochemistry:}

For immunohistochemistry, paraffin-embedded sections were pasted onto slides loaded with a solution of $2 \%$ silane in acetone. After dewaxing in xylene and rehydration in a reduced alcohol dilution series, the sections were boiled in a microwave oven in $0.02 \mathrm{M}$ citrate buffer at $\mathrm{pH}$ 6.0. After cooling, the sections were placed in $3 \%$ hydrogen peroxide and then washed twice in distilled water. After 30 min of incubation in $5 \%$ bovine serum albumin, the first antibody (diluted in $1 \%$ bovine serum) was used: rabbit polyclonal anti-human estrogen receptor alpha (ER $\alpha$ ) (amino acid 2-185 against ER $\alpha$, with 1:100 dilution of mouse monoclonal antihuman $\mathrm{P} 4$ receptor antibody added at the appropriate dilution) and incubated in a humidified chamber for $60 \mathrm{~min}$. Then, these sections were visualized using EnVision+System Horse Radish Peroxidase (HRP) method. In a further step, the sections were washed in tris(hydroxymethyl)aminomethane (TRIS) buffer and a chromogen solution of 3,3'-diaminobenzidine (DAB) prepared according to the manufacturer's instructions was added. In the final stage, sections were taken along a series of increasing concentrations of alcohol, xylene was exposed and mounted in Dibutylphthalate Polystyrene Xylene (DPX) medium.

\section{Integrated optical density (IOD):}

Microscopic analysis was performed under a BX40 vertical microscope equipped with a 5 megapixel (MP) DP50 3CCD peltier-cooled camera and an automatic image analysis system. All micrographs were collected under reference illumination using a 40-fold air microscope for histological examination. 
Micrographs were collected using LifeView software and automatically transmitted to microscopic Image 4.0 for analysis. The IOD of tissues with positive responses was measured from ten randomly selected target areas of interest (AOIs) per slide. DAB positive colors were sampled from $3 \times 3$ pixel regions and applied automatically to all AOIs. The average IOD from all 10 AOIs was taken as the IOD measurement for each slide.

\section{Morphometry:}

All morphometric measurements were performed using a fully automated BX40 vertical microscope (Olympus Poland, Warsaw, Poland) equipped with an electric stage, a high resolution 12 MP monochrome camera and Cell P (Olympus Poland, Warsaw, Poland) analysis software. Measurements were made under 10, 20, 40 or 100 times air microscopy under reference illumination conditions for histology. The diameter of ovarian follicles and Corpus Luteum (CL), thickness of endometrium, myometrium and serosa, height of superficial epithelium of endometrium, height of glandular epithelium of endometrium and area of endometrial section were measured.

\section{Statistical analysis:}

The results are given as mean \pm Standard Deviation (SD) and range in parentheses. Shapiro-Wilk test was used to assess the normality of data distribution and $\mathrm{t}$ test (unpaired samples) and Mann-Whitney $\mathrm{U}$ test were used to compare variables between groups. The homogeneity of group differences was assessed by the Brown-Forsythe test. The t-test and Wilcoxon signed rank test of paired samples were used to compare the variables between the uterine horn and the body in each group. All data were statistically analyzed using Statistical Package for the Social Sciences (SPSS) 23.0 software, all tests were two-tailed and $\mathrm{p}$ values below 0.05 were considered to indicate statistical significance.

\section{RESULTS AND DISCUSSION}

Before treatment, the mean serum P4 concentrations in the control group and the study group were 77.8 $\pm 7.0 \mathrm{nmol} / 1$ and $79.1 \pm 6.0 \mathrm{nmol} / \mathrm{l}$, respectively. Functional CL was present in both groups at the beginning of the experiment, as indicated by the basal circulating concentration of plasma P4 and morphological examination. $5 \mathrm{~d}$ after propofol injection, from 24 to $27 \mathrm{~d}$ after the $\mathrm{LH}$ peak, the $\mathrm{P} 4$ concentration in the study group began to decrease to lower than that in the control group $(\mathrm{p}<0.01)$ (Table 1).

Before propofol administration, the mean serum E concentration was relatively high $(123.4 \pm 25.0 \mathrm{pmol} / \mathrm{l})$, but gradually decreased to $88.2-99.1 \mathrm{pmol} / 1$ on the $27^{\text {th }}$ $\mathrm{d}$ after the peak of LH and remained approximately the same level thereafter. Throughout the study, there was no difference in serum E levels between the study and control female mice before and after administration of propofol (Table 2).

Large follicles (a small number of follicles in the ovary) were found only in study group mice. There was no significant difference in the number of medium-sized follicles $(0.6$ to $1.0 \mathrm{~mm})$ between the groups (Table 3 ). $\mathrm{CL}$ was found in all study female mice (9 in total) and

TABLE 1: COMPARISON OF SERUM PROGESTERONE CONCENTRATION IN TWO GROUPS

\begin{tabular}{lccccc}
\hline Parameter & LH after 8 d & 13 d & 18 d & 23 d & 27 d \\
\hline Research group & $56.7 \pm 23.2$ & $138.9 \pm 78.2$ & $87.4 \pm 34.2$ & $78.4 \pm 45.1$ & $39.2 \pm 13.2$ \\
Control group & $63.2 \pm 34.5$ & $126.7 \pm 94.3$ & $82.5 \pm 45.2$ & $81.3 \pm 46.2$ & $28.4 \pm 12.5$ \\
p value & $<0.01$ & $<0.01$ & $<0.01$ & $<0.01$ & $<0.01$ \\
\hline
\end{tabular}

TABLE 2: COMPARISON OF SERUM E LEVELS BETWEEN THE TWO GROUPS

\begin{tabular}{lccccc}
\hline Parameter & LH after 8 d & 13 d & 18 d & 23 d & 27 d \\
\hline Research group & $387.3 \pm 134.2$ & $343.2 \pm 132.5$ & $112.4 \pm 68.3$ & $134.2 \pm 87.2$ & $113.1 \pm 68.3$ \\
Control group & $153.2 \pm 135.3$ & $287.3 \pm 125.2$ & $154.2 \pm 85.3$ & $127.8 \pm 79.3$ & $124.1 \pm 68.3$ \\
p value & $<0.01$ & $<0.01$ & $<0.01$ & $<0.01$ & $<0.01$ \\
\hline
\end{tabular}

TABLE 3: COMPARISON OF HISTOMORPHOLOGICAL CHANGES OF OVARIAN STRUCTURE BETWEEN TWO GROUPS OF FEMALE MICE

\begin{tabular}{lcccccc}
\hline Parameter & $\mathrm{F} \mathrm{(<0.6)}$ & $\mathrm{F} \mathrm{(0.6-1.0)}$ & $\mathrm{F} \mathrm{(>1.0)}$ & $\mathrm{CL}(<0.6)$ & $\mathrm{CL}(\mathbf{0 . 6}-1.0)$ & $\mathrm{CL}(>1.0)$ \\
\hline Research group & - & $1.3 \pm 2.0$ & $0.7 \pm 1.2$ & 0 & $0.7 \pm 1.0$ & $6.5 \pm 2.4$ \\
Control group & - & $0.1 \pm 0.2$ & 0 & 0 & 0 & $4.7 \pm 1.4$ \\
p value & - & 0.23 & 0.51 & - & 0.34 & 0.18 \\
\hline
\end{tabular}

Note: Where F represents follicles 
in all controls ( 5 in total). No statistically significant difference was observed in the number of CLs ( 0.6 to $1.0 \mathrm{~mm}$ and $>1.0 \mathrm{~mm}$, respectively) between the two groups (Table 3).Compared with the control group, moderate CL was rare in the study group, but not in the control group.

Progesterone Receptor (PR) and peripheral blood sex hormone expression were detected in the ovaries of both control and study luteal phase mice. On d 26, propofol treatment did not alter PR and ER $\alpha$ expression (Table 4).

For gynecologists and patients of reproductive age, it is important to understand the potential changes in postoperative levels of female sex hormones after ovarian surgery ${ }^{[3]}$. One of the questions in this case is whether a specific anesthetic may have a greater impact on postoperative hormone levels. To our knowledge, our investigation is the first ${ }^{[2]}$. General anesthesia is used in routine surgery to block the transmission of sensory, motor and sympathetic nerves, leading to loss of consciousness, analgesia and suppression of autonomic reflexes. A variety of drugs are used for anesthesia in current practice and propofol is one of the most commonly used intravenous drugs administered by continuous infusion or intermittent bolus injection. Propofol is rapidly distributed to peripheral tissues including the central nervous system due to its high lipophilicity ${ }^{[4]}$. Interestingly, it is also found in ovarian follicular fluid. Serum propofol levels fluctuated during surgery, while follicular fluid levels steadily increased with increasing proportions. The dose of propofol suggested that the oocyte retrieval process should be as short as possible to minimize the accumulation of anesthetics in follicular fluid. These findings indicate that propofol accumulates in follicular fluid ${ }^{[5]}$. However, ovarian clearance of propofol has not been elucidated. In animal models, propofol has a dose and time dependent detrimental effect on oocyte maturation in vitro and embryo cleavage rate, in which case, residual propofol after surgery may affect ovarian function after surgery. To date, our current report is the first study to investigate sex hormone levels after surgery according to the use of the anesthetic propofol or not ${ }^{[16]}$. Marana et al. recently compared the levels of neuroendocrine stress hormones

TABLE 4: COMPARISON OF SEX HORMONE EXPRESSION IN PERIPHERAL BLOOD BETWEEN THE TWO GROUPS

\begin{tabular}{lcc}
\hline Parameter & PR & ERa \\
\hline Research group & $478.9 \pm 278.4$ & $819.3 \pm 231.2$ \\
Control group & $291.1 \pm 224.2$ & $718.2 \pm 332.4$ \\
p value & 0.213 & 0.587 \\
\hline
\end{tabular}

(including thyroid stimulating hormone and prolactin) during intravenous anesthesia with propofol and inhalation anesthesia. They found that prolactin levels were maintained for $4 \mathrm{~h}$ after surgery. When prolactin levels rise, the hypothalamus releases more dopamine, while elevated dopamine levels inhibit the release of gonadotropin-releasing hormone and $\mathrm{FSH}^{[13]}$. In another report, levels of stress hormones such as adrenaline and cortisol were found to be affected. In contrast to these studies, our study found no significant difference in E2 and FSH levels measured during the first postoperative menstrual cycle between the propofol and non-propofol groups. It can be inferred that the response of sex hormones to anesthetics may be different from that of neuroendocrine stress hormones ${ }^{[15]}$.

Aglepristone, a P4 antagonist, is used in clinical practice to treat pathologies caused by relatively prolonged exposure to high $\mathrm{P} 4$ levels, including cystic endometrial hyperplasia ${ }^{[4]}$. The peripheral blood P4 concentration in the first part of the luteal phase is high, similar to that found by Polisca et al. ${ }^{[3]}$. The gradual decrease in $\mathrm{P} 4$ concentration observed in mice given RU534 was similar to that observed in the control group, suggesting that this antiprogesterone triggered the expected physiological luteolysis process.

In his study, the decrease of P4 concentration in the study group began the $2^{\text {nd }} \mathrm{d}$ after RU534 administration and the P4 level on the $5^{\text {th }} \mathrm{d}$ was significantly lower than that in the control group. Ovarian ER $\alpha$ and PR were mainly found in CL in both groups, similar to the results of Parillo et al. According to their results, RU534 treatment was not associated with ER $\alpha$ and PR expression in the ovary ${ }^{[9]}$. To the author's knowledge, there is no literature describing the effects of RU534 on ovarian morphology and ER $\alpha$ and PR expression in mice. However, unlike rabbits, a slight difference was found, moderate CL in the study group was not statistically significant compared with no difference in the control group, but could serve as a clue to explain the premature luteolysis mechanism, which has not been confirmed in previous studies ${ }^{[6]}$.

Mifepristone (RU486), used to terminate human pregnancy, increases the peripheral concentration of Prostaglandin F2 alpha Metabolite (PGFM), the major metabolite of prostaglandin F2 alpha ${ }^{[6]}$. Another study suggested that early cessation of the luteal phase may be due to reduced LH pulse amplitude, LH pulse frequency and LH secretion during RU486 treatment. It is speculated that the action of this antiprogesterone compound at the hypothalamic-pituitary level may lead 
to premature luteolysis ${ }^{[3]}$. This may also be a reasonable explanation for the decrease in $\mathrm{P} 4$ concentration after taking RU534.

Detailed studies on the role of RU534 on the hypothalamic-pituitary-ovarian axis in dogs are lacking ${ }^{[11]}$. Progesterone inhibits the synthesis of ER $\alpha$ and $\mathrm{PR}^{[8]}$. In our study, the decrease in $\mathrm{P} 4$ concentration may have blocked this mechanism and indirectly led to higher expression of these receptors. In turn, this may increase tissue sensitivity to circulating $E$ in the blood. Thus, although no difference in E concentration between the two groups was observed, typical changes in estrus, such as hyperemia, thickening and inflammation caused by monocyte infiltration, could still be found in the endometrium of the study group.

In humans, propofol produces inactive metabolites. It is directly polymorphic O-glucuronidation in the human body to produce propofol-glucuronic acid, which is then hydroxylated to 2,6-diisopropyl-1,4-quinol. The latter substance then undergoes phase II metabolism, leading to the formation of other metabolites 4-(2,6-diisopropyl1,4-quinol) sulfate 1 and 4-(2,6-diisopropyl-1,4)glucuronic acid or sulfate ${ }^{[10]}$. Other minor phase I propofol metabolites such as 2-( $\omega$-propanol)-6isopropylphenol and 2-( $\omega$-propanol)-6-isopropyl-1,4quinol) are also described. Propofol is excreted from the urine after the glucose urea binding of the parent drug (to form propofol-glucuronic acid) and the sulfonyl and glucose urea binding of the hydroxylated metabolite to form 4-(2,6-diisopropyl-1,4-quinol) sulfate $1^{[6]}$. However, further studies are needed to elucidate and characterize polymorphic enzymes in explaining individual differences in glucuronidation metabolic pathways and their pharmacological and toxicological adverse effects.

Although positive associations of pharmacological genetic polymorphisms with good clinical significance have been found, the lack of reproducibility is a limitation because most studies focus on a single variant association and individual differences in propofol metabolism may be best explained by contributions from multiple pathways ${ }^{[3]}$. Indeed, the narrow therapeutic index and the significant difference in patient response to anesthesia and surgery make it more likely that serious adverse reactions will occur during the perioperative period. Other metabolites also need to be identified to confirm xenobiotic exposure in a wider detection window, especially in other samples ${ }^{[5]}$. To date, this mechanism has not been described in mice. There was no significant difference in the endometrium between the corners and the body between the groups. On the other hand, the thicker myometrium and higher PR expression in the uterine corpus compared with the angle of delivery may be due to the different functions of these parts of the uterus at delivery ${ }^{[10]}$. Significant thickening of the endometrium of the horn and body and the endometrium of the horn is likely to result from the concomitant activity of E and P4 and increased expression of their receptors. This mechanism develops when follicular cysts are present in the ovary and high levels of $\mathrm{P} 4$ in the pistil are parallel to E levels, ultimately leading to cystic endometrial hyperplasia ${ }^{[2]}$. However, the mechanism of action of RU534 described in this paper, including exacerbation of acute inflammatory response and infiltration of endometrial mononuclear immune cells, may accelerate the healing process of classical pustulosis and shorten the treatment time of chronic endometritis. In addition, these observations elucidate the mechanism of preventing implantation of fertilized eggs, as well as the contraceptive effect of RU534. After ovarian surgery, the ovarian reserve, the functional capacity of the ovary to provide oocytes, may decline. As described in this study, the most common ovarian reserve tests include measuring E2 and FSH levels in the early follicular phase ${ }^{[3]}$. Elevated levels of E2 in the early follicular phase (usually greater than 80 $\mathrm{pg} / \mathrm{ml}$ ) predicted that increased levels of E2 depleted by ovaries could inhibit pituitary FSH secretion, possibly masking increased levels of FSH, suggesting reduced ovarian reserve. High FSH levels indicate a decrease in fertility; however, FSH levels may vary due to different methods of FSH determination used in different laboratories and anti-Mullerian hormone (AMH) has been reported to decrease with reproductive ageing and is reported to recover after ovarian cystectomy and 3 mo after surgery ${ }^{[4]}$.

Overall, propofol was not different from other anesthetics in terms of ovarian function in female mice. The type of anesthetic did not seem to affect the levels of sex hormones in the peripheral blood of mice after surgery. Further prospective studies are needed to provide a comprehensive series of measurements of sex hormone levels.

\section{Author's contributions:}

Xiaoxia Qiu and Xianyuan Yi contributed equally to this work as co-first author.

\section{Conflicts of interest:}

The authors declared no conflict of interest. 


\section{REFERENCES}

1. Wang P, Chen J, Mu LH, Du QH, Niu XH, Zhang MY. Propofol inhibits invasion and enhances paclitaxel-induced apoptosis in ovarian cancer cells through the suppression of the transcription factor slug. Eur Rev Med Pharmacol Sci 2013;17(13):1722-9.

2. Eger EI. Characteristics of anesthetic agents used for induction and maintenance of general anesthesia. Am J Health Syst Pharm 2004;61(suppl_4):S3-10.

3. Ben-Shlomo I, Moskovich R, Golan J, Eyali V, Tabak A, Shalev E. The effect of propofol anaesthesia on oocyte fertilization and early embryo quality. Hum Reprod 2000;15(10):2197-9.

4. Su Z, Hou XK, Wen QP. Propofol induces apoptosis of epithelial ovarian cancer cells by upregulation of microRNA let-7i expression. Eur J Gynaecol Oncol 2014;35(6):688-91.

5. Urban BW, Bleckwenn M. Concepts and correlations relevant to general anaesthesia. Br J Anaesth 2002;89(1):3-16.

6. Marana E, Scambia G, Colicci S, Maviglia R, Maussier ML, Marana $\mathrm{R}$, et al. Leptin and perioperative neuroendocrine stress response with two different anaesthetic techniques. Acta Anaesthesiol Scand 2008;52(4):541-6.

7. Buyalos RP, Daneshmand S, Brzechffa PR. Basal estradiol and follicle-stimulating hormone predict fecundity in women of advanced reproductive age undergoing ovulation induction therapy. Fertil Steril 1997;68(2):272-7.

8. Chang HJ, Han SH, Lee JR, Jee BC, Lee BI, Suh CS, et al. Impact of laparoscopic cystectomy on ovarian reserve: serial changes of serum anti-Mullerian hormone levels. Fertil Steril 2010;94(1):343-9.

9. Marana E, Colicci S, Meo F, Marana R, Proietti R. Neuroendocrine stress response in gynecological laparoscopy:
TIVA with propofol versus sevoflurane anesthesia. J Clin Anesth 2010;22(4):250-5.

10. Parillo F, Dall'Aglio C, Brecchia G, Maranesi M, Polisca A, Boiti C, et al. Aglepristone (RU534) effects on luteal function of pseudopregnant rabbits: steroid receptors, enzymatic activities, and hormone productions in corpus luteum and uterus. Anim Reprod Sci 2013;138(1-2):118-32.

11. Jurka P, Max A, Hawrynska K, Snochowski M. Age-related pregnancy results and further examination of bitches after aglepristone treatment of pyometra. Reprod Domest Anim 2010;45(3):525-9.

12. Polisca A, Scotti L, Orlandi R, Brecchia G, Maranesi M, Zerani $\mathrm{M}$, et al. Aglepristone (RU534) administration to non-pregnant bitches in the mid-luteal phase induces early luteal regression. Theriogenology 2010;74(4):672-81.

13. Smotrich DB, Widra EA, Gindoff PR, Levy MJ, Hall JL, Stillman RJ. Prognostic value of day 3 estradiol on in vitro fertilization outcome. Fertil Steril 1995;64(6):1136-40.

14. Buyalos RP, Daneshmand S, Brzechffa PR. Basal estradiol and follicle-stimulating hormone predict fecundity in women of advanced reproductive age undergoing ovulation induction therapy. Fertil Steril 1997;68(2):272-7.

15. Marana E, Scambia G, Colicci S, Maviglia R, Maussier ML, Marana $\mathrm{R}$, et al. Leptin and perioperative neuroendocrine stress response with two different anaesthetic techniques. Acta Anaesthesiol Scand 2008;52(4):541-6.

16. Iwase A, Hirokawa W, Goto M, Takikawa S, Nagatomo Y, Nakahara $\mathrm{T}$, et al. Serum anti-Mullerian hormone level is a useful marker for evaluating the impact of laparoscopic cystectomy on ovarian reserve. Fertil Steril 2010;94(7) :2846-9. 